\title{
Antidepressant use and risk of suicide and attempted suicide or self harm in people aged 20 to 64: cohort study using a primary care database
}

\author{
Carol Coupland, ${ }^{1}$ Trevor Hill, ${ }^{1}$ Richard Morriss, ${ }^{2}$ Antony Arthur, ${ }^{3}$ Michael Moore, ${ }^{4}$ \\ Julia Hippisley-Cox ${ }^{1}$
}

${ }^{1}$ Division of Primary Care,

School of Medicine, University

of Nottingham, University Park,

Nottingham NG7 2RD, UK

2Institute of Mental Health, Jubilee Campus, Nottingham,

UK

${ }^{3}$ School of Health Sciences,

Faculty of Medicine and Health

Sciences, University of East

Anglia, Norwich, UK

4University of Southampton

Medical School, Primary Care

and Population Sciences,

Aldermoor Health Centre,

Southampton, UK

Correspondence to:

C Coupland carol.

coupland@nottingham.ac.uk

Additional material is

published online only. To

view please visit the journal

online (http://dx.doi.

org/10.1136/BMJ.h517)

Cite this as: $B M J$ 2015;350:h517 doi: 10.1136/bmj.h517

Accepted: 30 December 2014

\section{ABSTRACT}

OBJECTIVE

To assess the associations between different antidepressant treatments and the rates of suicide and attempted suicide or self harm in people with depression.

DESIGN

Cohort study.

SETTING

Patients registered with UK general practices contributing data to the QResearch database.

PARTICIPANTS

238963 patients aged 20 to 64 years with a first diagnosis of depression between 1 January 2000 and 31 July 2011, followed up until 1 August 2012.

\section{EXPOSURES}

Antidepressant class (tricyclic and related antidepressants, selective serotonin reuptake inhibitors, other antidepressants), dose, and duration of use, and commonly prescribed individual antidepressant drugs. Cox proportional hazards models were used to calculate hazard ratios adjusting for potential confounding variables.

\section{MAIN OUTCOME MEASURES}

Suicide and attempted suicide or self harm during follow-up.

RESULTS

During follow-up, $87.7 \%(n=209476)$ of the cohort received one or more prescriptions for antidepressants. The median duration of treatment was 221 days (interquartile range 79-590 days). During the first five years of follow-up 198 cases of suicide and

\section{WHAT IS ALREADY KNOWN ON THIS TOPIC \\ Depression is a common condition, and antidepressants are used extensively in its treatment \\ Rates of suicide and attempted suicide are higher in people with depression It is unclear to what extent suicide and attempted suicide rates vary when people with depression are treated with different antidepressants}

\section{WHAT THIS STUDY ADDS}

Rates of suicide and attempted suicide or self harm were similar during periods of treatment with selective serotonin reuptake inhibitors and tricyclic and related antidepressants overall, but were higher during periods of treatment with other antidepressants, although this may reflect indication biases and residual confounding

Suicide and self harm rates tend to be higher when antidepressants are started or stopped so the same care over risk assessment should be carried out when

treatment is stopped as when it is started
5243 cases of attempted suicide or self harm occurred. The difference in suicide rates during periods of treatment with tricyclic and related antidepressants compared with selective serotonin reuptake inhibitors was not significant (adjusted hazard ratio 0.84, 95\% confidence interval 0.47 to 1.50 ), but the suicide rate was significantly increased during periods of treatment with other antidepressants $(2.64,1.74$ to 3.99$)$. The hazard ratio for suicide was significantly increased for mirtazapine compared with citalopram $(3.70,2.00$ to 6.84). Absolute risks of suicide over one year ranged from $0.02 \%$ for amitriptyline to $0.19 \%$ for mirtazapine. There was no significant difference in the rate of attempted suicide or self harm with tricyclic antidepressants $(0.96,0.87$ to 1.08$)$ compared with selective serotonin reuptake inhibitors, but the rate of attempted suicide or self harm was significantly higher for other antidepressants $(1.80,1.61$ to 2.00$)$. The adjusted hazard ratios for attempted suicide or self harm were significantly increased for three of the most commonly prescribed drugs compared with citalopram: venlafaxine $(1.85,1.61$ to 2.13$)$, trazodone $(1.73,1.26$ to 2.37$)$, and mirtazapine $(1.70,1.44$ to 2.02), and significantly reduced for amitriptyline $(0.71,0.59$ to 0.85$)$. The absolute risks of attempted suicide or self harm over one year ranged from $1.02 \%$ for amitriptyline to $2.96 \%$ for venlafaxine. Rates were highest in the first 28 days after starting treatment and remained increased in the first 28 days after stopping treatment.

\section{CONCLUSION}

Rates of suicide and attempted suicide or self harm were similar during periods of treatment with selective serotonin reuptake inhibitors and tricyclic and related antidepressants. Mirtazapine, venlafaxine, and trazodone were associated with the highest rates of suicide and attempted suicide or self harm, but the number of suicide events was small leading to imprecise estimates. As this is an observational study the findings may reflect indication biases and residual confounding from severity of depression and differing characteristics of patients prescribed these drugs. The increased rates in the first 28 days of starting and stopping antidepressants emphasise the need for careful monitoring of patients during these periods.

\section{Introduction}

Rates of suicide and self harm are greatly increased in people with depression ${ }^{12}$ and reduction of these risks is a major consideration when treating such patients. Paradoxically, although antidepressants have been shown 
to be effective in reducing the symptoms of depression $^{34}$ there is concern that rates of suicide and self harm may actually be increased by treatment, particularly in younger people. ${ }^{56}$ A meta-analysis of 372 randomised placebo controlled trials of antidepressants found that among adults aged less than 25 the risk of suicidal behaviour was increased during treatment with antidepressants, whereas no association was found in adults aged 25 to 64, and in those aged 65 or more the risk was reduced. ${ }^{7}$ These findings were supported by a meta-analysis of eight observational studies involving more than 200000 patients, ${ }^{8}$ which found an increased risk of suicide among adolescents treated with selective serotonin reuptake inhibitors compared with no antidepressant treatment, but a reduced risk among adults.

The meta-analysis by Stone ${ }^{7}$ indicated possible differences in risk of suicidal behaviour between different antidepressants, but these findings were based on a small number of events. In a cohort study, venlafaxine was associated with an increased risk of suicide and attempted suicide compared with three other antidepressants, ${ }^{9}$ although the authors concluded that this may have been due to residual confounding. In another cohort study the reported rates of suicide and attempted suicide were similar for different antidepressants, except for a higher rate of suicidal acts in users of venlafaxine compared with selective serotonin reuptake inhibitors, but this association was reduced in secondary analyses. ${ }^{10}$ In a cohort study of adults aged 65 or more with depression the highest rates of attempted suicide or self harm were in those treated with venlafaxine, mirtazapine, or trazodone. ${ }^{11}$

Uncertainty remains about the risks of suicide and self harm for different antidepressants and whether these risks vary by dose and duration of use. This is particularly important to determine, now that suicide rates in the United Kingdom and many other countries have started to increase after a period when they were decreasing. ${ }^{12-14}$ We carried out a cohort study in people aged 20 to 64 to investigate the associations between different antidepressants and the risk of suicide and attempted suicide or self harm.

\section{Methods}

This cohort study was designed to assess the associations of antidepressant treatment and several different adverse outcomes, including suicide and attempted suicide or self harm. Full details of the study design and methods are in the study protocol. ${ }^{15}$

\section{Study cohort}

The study cohort was selected from a large primary care database (QResearch, version 34). QResearch is derived from the anonymised health records of over 12 million patients from more than 600 general practices across the United Kingdom. The practices record data using the Egton Medical Information Systems medical records system. Recorded information includes data on patients' diagnoses, symptoms, consultations, referrals, test results, and prescriptions. The database is linked at patient level to Office for National Statistics mortality data.

The study sample comprised an open cohort of patients with a first recorded diagnosis of depression between 1 January 2000 and 31 July 2011, at age 20 to 64 . We used diagnostic Read codes to identify patients with a diagnosis of depression, using case definitions that have been used in previous studies. ${ }^{11} 1617$ Patients were eligible for inclusion only if their diagnosis occurred at least 12 months after registration with a study practice and the date of the installation of the practice computer system. Patients were eligible for inclusion regardless of whether they received prescriptions for antidepressants. To reduce the risk of indication bias, we considered patients who received prescriptions for antidepressants but did not have a recorded diagnosis of depression to be ineligible, as the prescriptions may have been for conditions other than depression.

We excluded patients from the cohort if they had a previous recorded diagnosis of depression; were temporary residents; had a diagnosis of schizophrenia, bipolar disorder, or other type of psychosis; or had been prescribed lithium or antimanic drugs at the study entry date. Patients were also excluded if they had received prescriptions for an antidepressant either before the study start date (1 January 2000), before their date of registration with the practice (if later), before the age of 20, or more than 36 months before their first recorded diagnosis of depression.

We defined the date of entry into the study (index date) as the date of the first recorded diagnosis of depression, or the date of the first prescription for an antidepressant if earlier. Participants were followed up until the earliest of date of death, date of leaving the practice, or the end of follow-up (1 August 2012). Most analyses were restricted to the first five years of follow-up.

\section{Outcomes}

We identified suicides during follow-up as patients with either a code for suicide or an open verdict on their linked death certificate (using international classification of diseases codes, ninth and 10th revisions) or patients with a Read code in their medical record for attempted suicide or self harm who died within 30 days. We identified cases of attempted suicide or self harm from the patients' medical records, based on Read codes used in other studies. ${ }^{91618}$ We only considered the first attempted suicide or self harm event in the analysis of this outcome, and excluded patients with a previous attempted suicide or self harm event recorded at baseline.

\section{Exposure data}

We extracted details of all individual prescriptions for antidepressants during follow-up, including the issue date, the type and dose of antidepressant, dosage instructions, and quantity of tablets prescribed. To determine the duration of each prescription in days we divided the number of tablets prescribed by the dosing instructions (for example, number of tablets to be taken 
each day). Where the number of tablets prescribed was recorded but dosing instructions were missing or not sufficiently detailed ( $<5 \%$ of prescriptions) we used an assumed duration, taking account of the number of tablets prescribed as in our previous study. ${ }^{19}$ We calculated the daily dose of each prescription by multiplying the specified dose of each tablet by the number of tablets to be taken each day. Prescriptions for the same drug issued on the same day were counted as a single prescription and the doses combined.

For the main analyses we grouped antidepressants according to the major classes as given in the British National Formulary-namely, tricyclic and related antidepressants (section 4.3.1), selective serotonin reuptake inhibitors (section 4.3.3), monoamine oxidase inhibitors (section 4.3.2), and other antidepressants (section 4.3.4). Where patients received prescriptions for different drugs within a class or for drugs from different classes on the same date we classified these as combined prescriptions.

To enable a comparison of dose between the antidepressant classes, for each prescription we converted the prescribed daily dose to a number of defined daily doses, using the values assigned by the World Health Organization's Collaborating Centre for Drug Statistics Methodology (www.whocc.no/atc_ddd_index). WHO's definition of a defined daily dose is the assumed average maintenance dose each day for a drug used for its main indication in adults (for example, the defined daily dose for citalopram is $20 \mathrm{mg}$, for fluoxetine is $20 \mathrm{mg}$, and for amitriptyline is $75 \mathrm{mg}$ ), although this may not necessarily reflect the recommended dose, and individual patients may be prescribed higher or lower doses.

Where numbers were sufficient, we also assessed individual antidepressants. As in our previous study, we examined the 11 most commonly prescribed individual antidepressants separately. ${ }^{11}$

\section{Confounding variables}

We considered as confounding variables those likely to be associated with the risk of suicide or attempted suicide or self harm, or with the likelihood of receiving treatment for different antidepressants, based on variables included in our previous study of antidepressants in people aged 65 or more..$^{11}$

Confounders were age at study entry date, sex, year of diagnosis of depression, severity of index diagnosis of depression (categorised as mild, moderate, or severe based on the Read code for the index diagnosis, using codes published by Martinez and colleagues ${ }^{16}$ and some additional classification by a member of the study team), deprivation (Townsend deprivation score corresponding to the patient's postcode, in fifths), smoking status (non-smoker, former smoker, light smoker (1-9 cigarettes/day), moderate smoker (10-19 cigarettes/day), heavy smoker ( $\geq 20$ cigarettes/day), not recorded), alcohol intake (none, trivial ( $<1$ UK unit/day), light (1-2 units/day), medium (3-6 units/day,) heavy (7-9 units/day,) very heavy ( $>9$ units/day), not recorded), ethnic group (categorised as white/not recorded or non-white (Indian, Pakistani, Bangladeshi, other Asian, black African, black Caribbean, Chinese, other including mixed)), comorbidities at baseline (coronary heart disease, diabetes, hypertension, cancer, epilepsy or seizures, hypothyroidism, osteoarthritis, asthma or chronic obstructive airways disease), and use of other drugs at baseline (statins, non-steroidal anti-inflammatory drugs, aspirin, antihypertensive drugs, anticonvulsants, hypnotics or anxiolytics, oral contraceptives, hormone replacement therapy). In addition, the analysis of suicide as an outcome included attempted suicide or self harm at baseline as a confounding variable. In the analysis of attempted suicide or self harm we also adjusted for stroke or transient ischaemic attack, rheumatoid arthritis, osteoporosis, liver disease, renal disease, obsessive-compulsive disorder, antipsychotics, bisphosphonates, and anticoagulants. These were not included in the analysis of suicide because of the smaller number of events, and the prevalence of these confounders was less than $1 \%$ in the study cohort.

\section{Statistical analysis}

We used Cox's proportional hazards models to assess the associations between exposure to antidepressant drugs and the two outcomes (suicide and attempted suicide or self harm). To account for patients starting and stopping treatment during follow-up and also changing between treatments, we treated antidepressant exposure as a time varying exposure. We considered patients to have been exposed to a drug if there were no gaps of more than 90 days between the end of one prescription and the start of the next. In the analysis of attempted suicide or self harm we excluded patients who already had the outcome recorded at baseline. The main analyses were based on the first five years of follow-up after the study entry date, by censoring any follow-up after this date. We used robust standard errors to account for clustering of patients within practices. ${ }^{20}$

In the analysis we calculated unadjusted and adjusted analysis hazard ratios for antidepressant class, by comparing current treatment using tricyclic and related antidepressants, other antidepressants, and combined antidepressants with treatment using selective serotonin reuptake inhibitors. We included a category of "no current treatment" for periods of time where patients were not treated with antidepressants, which also included follow-up time for patients who were not prescribed any antidepressants during follow-up. The number of patients prescribed monoamine oxidase inhibitors was too small for this group to be analysed, so we excluded from the analyses those patients prescribed monoamine oxidase inhibitors at any time.

Analyses were also carried out for dose categories within each antidepressant class $(\leq 0.5,>0.5$ and $\leq 1.0$, and $>1.0$ defined daily doses) using the middle dose category for selective serotonin reuptake inhibitors as the reference group, and we carried out tests for trend using the doses within each class as continuous variables. We analysed duration of antidepressant use and time since 
stopping within each antidepressant class as a time varying exposure (categorised as no use (reference group), 1-28 days, 29-84 days, and $\geq 85$ days after starting treatment, and 1-28 days, 29-84 days, and 85-182 days after stopping treatment). We only allocated follow-up time to the time since stopping treatment categories if patients did not switch directly to another antidepressant, otherwise we allocated their follow-up time to time since starting categories for the new drug. Analyses were carried out for the 11 most commonly prescribed antidepressants, with the most commonly prescribed antidepressant (citalopram) as the reference category.

To determine whether significant differences existed between the antidepressant classes and between the individual drugs, we carried out Wald's tests. We tested for interactions between drug class and age (continuous) and carried out subgroup analyses split by age band (20-29, 30-39, 40-49, and 50-64 years). We assessed the proportional hazards assumption using log minus log plots.

As a sensitivity analysis we repeated the analyses restricted to patients who received at least one prescription for an antidepressant during follow-up, excluding untreated patients. We carried out this analysis because patients who were untreated during follow-up might differ from treated patients in ways that would be difficult to account for in the analysis (such as having a dislike of tablets, a preference for non-drug treatments, or less severe depression), so we carried out this sensitivity analysis to test whether our results were robust to exclusion of these patients. We also carried out an analysis restricted to the first year of follow-up and a further analysis including all outcome events that occurred within the entire follow-up period. These analyses were done to assess whether any associations between antidepressant drugs and the risks of suicide and attempted suicide or self harm for five years' follow-up were also present in analyses over one year and over the entire follow-up period. In particular the baseline characteristics are less likely to change during one year, and fewer switches occur between different antidepressant drugs, so the results from the one year analysis are less likely to be influenced by residual confounding. We performed analyses for the entire follow-up since these have increased power and include long durations of use. In an additional analysis we used a minimum gap of 30 days between the end of one prescription and the start of the next rather than 90 days to classify periods of non-use.
We carried out a further analysis of the suicide outcome restricted to deaths specifically coded as suicide on the linked death certificate and not including open verdicts or suicides only identified from the medical records.

We calculated absolute risks of both outcomes over one year, accounting for the confounding variables using adjusted hazard ratios from the analysis for one year of follow-up based on the method described by Altman et al. ${ }^{21}$

To maximise power we included all eligible patients from the database in the analyses. We used a P value of less than 0.01 (two tailed) to determine statistical significance. Analyses were carried out using Stata (v12.1).

\section{Results}

From 1 January 2000 to 31 July 2011, 327235 patients had a first diagnosis of depression between the ages of 20 and 64. A total of 88272 (27.0\%) patients were excluded from the study cohort because they had schizophrenia, bipolar disorder, or other psychoses or had been prescribed lithium or antimanic drugs (7152 patients, 2.2\%) and/or had been prescribed an antidepressant before the study entry date aged less than 20 , or more than 36 months before the recorded date of depression $(83824$ patients, 25.6\%); 2704 patients met both exclusion criteria. This left 238963 patients from 687 practices in the study cohort (fig 1).

Table 1 shows the baseline characteristics of the study cohort. The mean age was 39.5 (SD 11.1) years, and there were 92935 men (38.9\%) and 146028 (61.1\%) women. The total number of person years of follow-up was 1307326 , with a median of 5.2 (interquartile range 2.5-8.2) years.

\section{Antidepressant treatment during follow-up}

During follow-up, $87.7 \%$ of patients $(n=209476)$ received one or more prescriptions for an antidepressant, with 3337336 prescriptions for antidepressants received in total; 29708 patients (14.2\%) only received a single prescription during follow-up. The median duration of treatment with antidepressants during follow-up was 221 (interquartile range 79-590) days, with $36.6 \%$ of treated patients having one or more years of treatment and $5.5 \%$ having five or more years.

Selective serotonin reuptake inhibitors were the most commonly prescribed drug class, comprising 71.3\% (2379668) of prescriptions for antidepressants; there were 533798 prescriptions (16.0\%) for tricyclic and related antidepressants and $422079(12.7 \%)$ for the

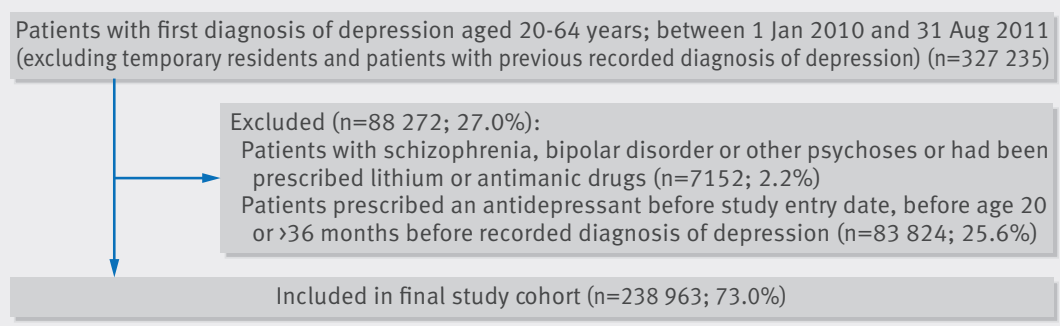

Fig 1 | Flow chart showing selection of patients included in study cohort. 2704 patients met both exclusion criteria 


\begin{tabular}{|c|c|}
\hline Characteristics & $\begin{array}{l}\text { Study cohort } \\
(n=238963)\end{array}$ \\
\hline Men & $92935(38.9)$ \\
\hline Women & $146028(61.1)$ \\
\hline \multicolumn{2}{|l|}{ Age (years): } \\
\hline $20-29$ & $51212(21.4)$ \\
\hline $30-39$ & $77141(32.3)$ \\
\hline $40-49$ & $59260(24.8)$ \\
\hline $50-59$ & $39573(16.6)$ \\
\hline $60-64$ & $11777(4.9)$ \\
\hline Mean (SD) age (years) & $39.5(11.1)$ \\
\hline \multicolumn{2}{|l|}{ Ethnic group: } \\
\hline White/not recorded & $227451(95.2)$ \\
\hline Indian & $1922(0.8)$ \\
\hline Pakistani & $1714(0.7)$ \\
\hline Bangladeshi & $1000(0.4)$ \\
\hline Other Asian & $991(0.4)$ \\
\hline Caribbean & $1520(0.6)$ \\
\hline Black African & $1386(0.6)$ \\
\hline Chinese & $307(0.1)$ \\
\hline Other & $2672(1.1)$ \\
\hline \multicolumn{2}{|l|}{ Depression severity (index diagnosis): } \\
\hline Mild & $171208(71.7)$ \\
\hline Moderate & $59140(24.8)$ \\
\hline Severe & $8615(3.6)$ \\
\hline \multicolumn{2}{|l|}{ Smoking status: ${ }^{*}$} \\
\hline Recorded & $233290(97.6)$ \\
\hline Non-smoker & $110849(47.5)$ \\
\hline Former smoker & $35132(15.1)$ \\
\hline Light smoker (1-9 cigarettes/day) & $24104(10.3)$ \\
\hline Moderate smoker (10-19 cigarettes/day) & $40546(17.4)$ \\
\hline Heavy smoker ( $\geq 20$ cigarettes/day) & $22659(9.7)$ \\
\hline \multicolumn{2}{|l|}{ Alcohol consumption:* } \\
\hline Recorded & $203189(85.0)$ \\
\hline Non-drinker & $55253(27.2)$ \\
\hline Trivial (<1 UK unit/day) & $77579(38.2)$ \\
\hline Light (1-2 units/day) & $51310(25.3)$ \\
\hline Moderate (3-6 units/day) & $14482(7.1)$ \\
\hline Heavy (7-9 units/day) & $2174(1.1)$ \\
\hline Very heavy ( $>9$ units/day) & $2391(1.2)$ \\
\hline \multicolumn{2}{|l|}{ Townsend deprivation fifth: ${ }^{*}$} \\
\hline Recorded & $230762(96.6)$ \\
\hline 1 (least deprived) & $45021(19.5)$ \\
\hline 2 & $46207(20.0)$ \\
\hline 3 & $48293(20.9)$ \\
\hline 4 & $47063(20.4)$ \\
\hline 5 (most deprived) & $44178(19.1)$ \\
\hline \multicolumn{2}{|l|}{ Comorbidities: } \\
\hline Coronary heart disease & $4109(1.7)$ \\
\hline Diabetes & $7371(3.1)$ \\
\hline Hypertension & $17217(7.2)$ \\
\hline Stroke or transient ischaemic attack & $1741(0.7)$ \\
\hline Asthma or chronic obstructive airways disease & $31816(13.3)$ \\
\hline Cancer & $3810(1.6)$ \\
\hline Epilepsy or seizures & $3325(1.4)$ \\
\hline Hypothyroidism & $5267(2.2)$ \\
\hline Obsessive-compulsive disorder & $494(0.2)$ \\
\hline Rheumatoid arthritis & $1301(0.5)$ \\
\hline Osteoarthritis & $7228(3.0)$ \\
\hline Osteoporosis & $867(0.4)$ \\
\hline Liver disease & $698(0.3)$ \\
\hline \multirow[t]{2}{*}{ Renal disease } & $549(0.2)$ \\
\hline & Continue \\
\hline
\end{tabular}

Table 1 (continued) | Characteristics of study cohort at baseline. Values are numbers (percentages) unless stated otherwise

\begin{tabular}{ll}
\hline Previous attempted suicide/self harm & $10174(4.3)$ \\
\hline Drugs at baseline: & \\
\hline Anticonvulsants & $2672(1.1)$ \\
\hline Antihypertensives & $25344(10.6)$ \\
\hline Antipsychotics & $836(0.4)$ \\
\hline Anticoagulants & $1073(0.5)$ \\
\hline Aspirin & $7159(3.0)$ \\
\hline Bisphosphonates & $854(0.4)$ \\
\hline Hypnotics or anxiolytics & $11354(4.8)$ \\
\hline Non-steroidal anti-inflammatory drugs & $12725(5.3)$ \\
\hline Statins & $10823(4.5)$ \\
\hline Oral contraceptives $\dagger$ & $27396(18.8)$ \\
\hline Hormone replacement therapy† & $7207(4.9)$ \\
\hline $\begin{array}{l}\text { *Percentages out of total of recorded values. } \\
\text { tWomen only. }\end{array}$
\end{tabular}

group of other antidepressants. Monoamine oxidase inhibitors were the least commonly prescribed class, comprising only 1791 prescriptions (0.05\%) in 156 patients. There were 83784 combined prescriptions where two or more different antidepressant drugs were prescribed on the same day.

Figure 2 shows the number of prescriptions during follow-up for the 11 most commonly prescribed antidepressants, which comprised $97.7 \%$ of all prescriptions for antidepressants. Citalopram was the most commonly prescribed antidepressant (1023255 prescriptions, $31.5 \%$ of total). Citalopram and fluoxetine accounted for $77.6 \%$ of prescriptions for selective serotonin reuptake inhibitors, amitriptyline and dosulepin for $77.3 \%$ of prescriptions for tricyclic and related antidepressants, and venlafaxine and mirtazapine for $90.7 \%$ of prescriptions for other antidepressants. Supplementary tables 1s to 3s show the distributions of baseline characteristics according to the first antidepressant prescribed for these 11 drugs.

\section{Associations with suicide}

After omitting the patients with prescriptions for monoamine oxidase inhibitors, there were 238807 patients in the study cohort. During the first five years of follow-up 198 cases of suicide occurred in this study cohort, giving

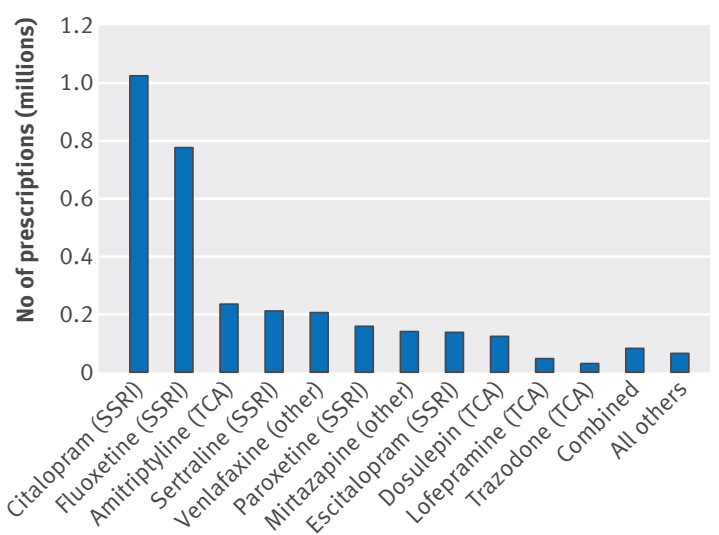
follow-up for most commonly prescribed antidepressants 
an incidence rate of 22 per 100000 person years (43 per 100000 in men and 9 per 100000 in women). Of these suicide cases, 156 were only identified from the linked death records, 18 only from primary care records, and 24 from both sources. Among the 180 cases identified from linked death records, 48 were coded as open verdicts.

Suicide rates did not differ significantly when comparing periods of tricyclic antidepressant treatment with selective serotonin reuptake inhibitor treatment (adjusted hazard ratio $0.84,95 \%$ confidence interval 0.47 to 1.50 ), but rates increased significantly during periods of treatment with the other antidepressants compared with selective serotonin reuptake inhibitors $(2.64,1.74$ to 3.99, table 2). Adjusted hazard ratios tended to increase with dose for selective serotonin reuptake inhibitors $(\mathrm{P}=0.02)$, but not for tricyclic antidepressants $(\mathrm{P}=0.6)$ or other antidepressants $(\mathrm{P}=0.9)$ (table 2).

In the analysis of the 11 most commonly prescribed drugs, the adjusted hazard ratio was significantly increased (at $\mathrm{P}<0.01$ ) for mirtazapine compared with citalopram (3.70, 2.00 to 6.84). There was also some indication of an increased risk for venlafaxine compared with citalopram $(2.23,1.14$ to $4.39, \mathrm{P}=0.02)$.

Hazard ratios were highest in the first 28 days after starting treatment for tricyclic antidepressants, selective serotonin reuptake inhibitors, and, particularly, for other antidepressants, and also in the first 28 days after stopping treatment (fig 3).

\begin{tabular}{|c|c|c|c|c|c|c|}
\hline \multirow[b]{2}{*}{ Variables } & \multicolumn{3}{|c|}{ Unadjusted analysis } & \multirow[b]{2}{*}{$P$ value } & \multicolumn{2}{|l|}{ Adjusted analysis* } \\
\hline & No of eventst & Person years $t$ & Hazard ratio $(95 \% \mathrm{Cl})$ & & Hazard ratio $(95 \% \mathrm{Cl})$ & Pvalue \\
\hline \multicolumn{7}{|l|}{ Antidepressant class } \\
\hline SSRIS & 79 & 228233 & 1.00 & & 1.00 & \\
\hline TCAs & 13 & 41807 & 0.90 (0.51 to 1.62$)$ & 0.7 & 0.84 (0.47 to 1.50$)$ & 0.6 \\
\hline Other antidepressants & 31 & 28487 & $3.62(2.41$ to 5.44$)$ & $<0.001$ & 2.64 (1.74 to 3.99 ) & $<0.001$ \\
\hline Combined antidepressants & 2 & 4299 & $1.62(0.39$ to 6.70$)$ & 0.5 & $1.36(0.32$ to 5.68$)$ & 0.7 \\
\hline No current use & 69 & 575623 & $0.45(0.32$ to 0.62$)$ & $<0.001$ & $0.39(0.28$ to 0.55$)$ & $<0.001$ \\
\hline \multicolumn{7}{|c|}{ Antidepressant class and dose categories (defined daily doses) $\ddagger$} \\
\hline \multicolumn{7}{|l|}{ SSRIS: } \\
\hline$\leq 0.5$ & 1 & 16289 & $0.20(0.03$ to 1.41$)$ & 0.1 & 0.22 (0.03 to 1.60$)$ & 0.1 \\
\hline$>0.5 / \leq 1.0$ & 55 & 159847 & 1.00 & & 1.00 & \\
\hline$>1.0$ & 21 & 43226 & 1.74 (1.07 to 2.81$)$ & 0.02 & 1.55 (0.95 to 2.53$)$ & 0.08 \\
\hline Test for trend§ & & & & 0.002 & & 0.02 \\
\hline \multicolumn{7}{|l|}{ TCAs: } \\
\hline$\leq 0.5$ & 6 & 23895 & 0.76 (0.33 to 1.75$)$ & 0.5 & 0.74 (0.32 to 1.72$)$ & 0.5 \\
\hline$>0.5 / \leq 1.0$ & 3 & 8502 & $1.14(0.36$ to 3.64$)$ & 0.8 & $0.95(0.30$ to 3.01$)$ & 0.9 \\
\hline$>1.0$ & 3 & 5363 & $1.78(0.56$ to 5.69$)$ & 0.3 & $1.46(0.46$ to 4.66$)$ & 0.5 \\
\hline Test for trend§ & & & & 0.3 & & 0.6 \\
\hline \multicolumn{7}{|l|}{ Others: } \\
\hline$\leq 0.5$ & 5 & 4100 & 4.09 (1.65 to 10.13$)$ & 0.002 & 3.44 (1.38 to 8.61$)$ & 0.008 \\
\hline$>0.5 / \leq 1.0$ & 18 & 13404 & 4.73 (2.81 to 7.97$)$ & $<0.001$ & 3.36 (1.98 to 5.70$)$ & $<0.001$ \\
\hline$>1.0$ & 7 & 8529 & 3.19 (1.44 to 7.06$)$ & 0.004 & 2.15 (0.96 to 4.83$)$ & 0.06 \\
\hline Test for trend§ & & & & 0.9 & & 0.9 \\
\hline \multicolumn{7}{|l|}{ Antidepressant drugs } \\
\hline \multicolumn{7}{|l|}{ SSRIS: } \\
\hline Citalopram & 30 & 95640 & 1.00 & & 1.00 & \\
\hline Escitalopram & 4 & 13539 & 0.93 (0.32 to 2.64$)$ & 0.9 & 0.89 (0.31 to 2.55$)$ & 0.8 \\
\hline Fluoxetine & 27 & 82935 & 0.96 (0.56 to 1.65$)$ & 0.9 & 1.03 (0.60 to 1.79$)$ & 0.9 \\
\hline Paroxetine & 11 & 16705 & 1.95 (0.96 to 3.95$)$ & 0.06 & 1.59 (0.77 to 3.28$)$ & 0.2 \\
\hline Sertraline & 7 & 19139 & 1.15 (0.51 to 2.59) & 0.7 & 1.15 (0.51 to 2.63 ) & 0.7 \\
\hline \multicolumn{7}{|l|}{ TCAs: } \\
\hline Amitriptyline & 4 & 19845 & 0.64 (0.22 to 1.82$)$ & 0.4 & 0.68 (0.24 to 1.96$)$ & 0.5 \\
\hline Dosulepin & 5 & 12292 & 1.22 (0.47 to 3.17$)$ & 0.7 & 1.04 (0.40 to 2.74$)$ & 0.9 \\
\hline Lofepramine & 2 & 4863 & 1.20 (0.29 to 5.03$)$ & 0.8 & $1.06(0.25$ to 4.60$)$ & 0.9 \\
\hline Trazodone & 2 & 2390 & 2.69 (0.64 to 11.28$)$ & 0.2 & 2.00 (0.47 to 8.57$)$ & 0.3 \\
\hline \multicolumn{7}{|l|}{ Others: } \\
\hline Mirtazapine & 16 & 10343 & 5.18 (2.84 to 9.43 ) & $<0.001$ & $3.70(2.00$ to 6.84$)$ & $<0.001$ \\
\hline Venlafaxine & 13 & 15835 & 3.09 (1.62 to 5.91$)$ & 0.001 & 2.23 (1.14 to 4.39$)$ & 0.02 \\
\hline All other antidepressants & 2 & 5001 & 1.31 (0.32 to 5.32$)$ & 0.7 & $1.15(0.28$ to 4.76$)$ & 0.8 \\
\hline Combined antidepressants & 2 & 4299 & $1.72(0.40$ to 7.44$)$ & 0.5 & 1.45 (0.33 to 6.43$)$ & 0.6 \\
\hline
\end{tabular}

SSRIs=selective serotonin reuptake inhibitors; TCAs=tricyclic and related antidepressants.

*Adjusted for age, sex, year of diagnosis of depression, severity of depression, deprivation, smoking status, alcohol intake, ethnic group, attempted suicide or self harm at baseline, coronary

heart disease, diabetes, hypertension, cancer, epilepsy or seizures, hypothyroidism, osteoarthritis, asthma or chronic obstructive airways disease, statins, non-steroidal anti-inflammatory

drugs, aspirin, antihypertensives, anticonvulsants, hypnotics or anxiolytics, oral contraceptives, and hormone replacement therapy.

tBased on numbers in adjusted analysis.

†Total numbers of events in analysis of dose are reduced owing to missing data on dose.

$\S$ Test for trend uses continuous values of dose. 


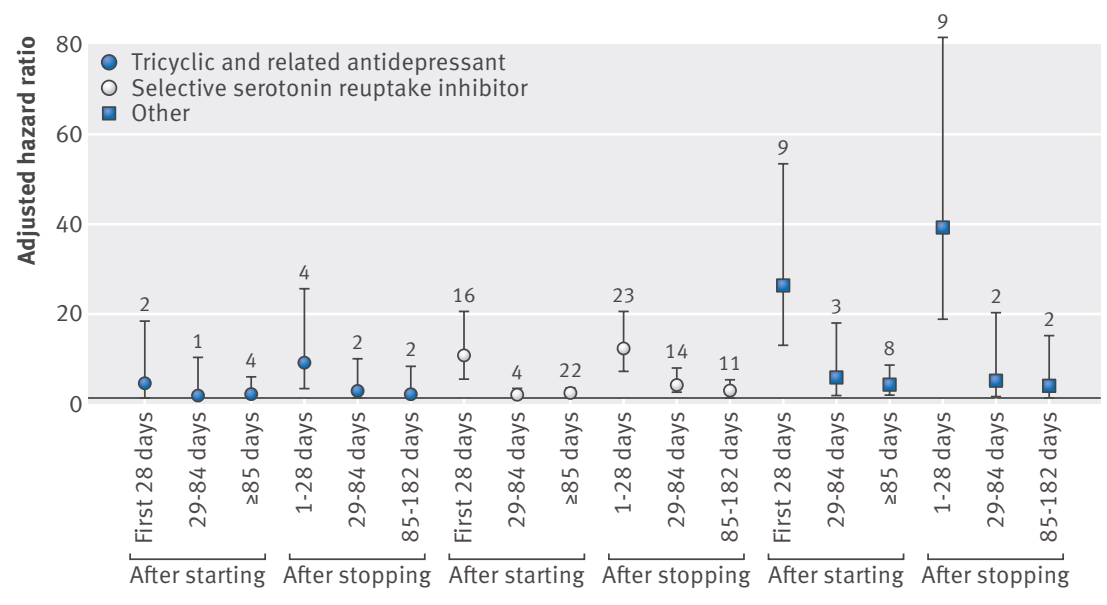

Time since starting and stopping treatment

Fig 3 | Adjusted hazards and $95 \%$ confidence intervals for suicide according to time since starting and stopping different classes of antidepressant compared with untreated periods. Numbers of events are included

Hazard ratios changed little when patients who had not received any antidepressant prescriptions during follow-up were removed from the analysis. Hazard ratios were also generally similar when either just the first year of follow-up (see supplementary table 4s) or the entire follow-up period was used in the analysis, or when a 30 day gap was used to define unexposed periods (see supplementary table 6s). Hazard ratios tended to be higher, although confidence intervals were wider, when the analysis was restricted to the 132 cases with specific codes for suicide on the linked death certificate (see supplementary table 7s).

\section{Associations with attempted suicide or self harm} At baseline 10174 patients (4.3\%) had a record of attempted suicide or self harm. We omitted these patients from the analyses of attempted suicide or self harm, and also those with prescriptions for monoamine oxidase inhibitors, leaving 228643 in the study cohort. During the first five years of follow-up there were 5243 cases of attempted suicide or self harm, giving an incidence rate of 613 per 100000 person years (737 per 100000 in men and 517 per 100000 in women).

The rates of attempted suicide or self harm did not differ significantly for tricyclic antidepressants compared with selective serotonin reuptake inhibitors $(0.96$, 0.87 to 1.08 ) but hazard ratios were significantly increased during periods of treatment with other antidepressants $(1.80,1.61$ to 2.00$)$ or with combined prescriptions (2.00, 1.54 to 2.59 , table 3 ). In analyses of dose, adjusted hazard ratios increased significantly with dose for selective serotonin reuptake inhibitors and other antidepressants (both $\mathrm{P}<0.001$ ) and also tended to increase with dose for tricyclic antidepressants $(\mathrm{P}=0.013)$ (table 3$)$.

Hazard ratios were significantly increased for three of the most commonly prescribed drugs compared with citalopram (table 3); venlafaxine (adjusted hazard ratio 1.85 , 95\% confidence interval 1.61 to 2.13 ), trazodone $(1.73,1.26$ to 2.37$)$, and mirtazapine (1.70,
1.44 to 2.02). The hazard ratio for amitriptyline compared with citalopram was significantly reduced $(0.71$, 0.59 to 0.85 ).

Rates of attempted suicide or self harm were highest in the first 28 days after starting treatment, particularly for other antidepressants (fig 4).

Hazard ratios were slightly reduced when patients who had not received any prescriptions for antidepressants during follow-up were removed from the analysis. Results were generally similar when either just the first year of follow-up (see supplementary table 5s) or the entire follow-up period was used in the analysis, and also were similar when a 30 day gap was used to define unexposed periods (see supplementary table 7s).

\section{Interactions with age}

Overall there was no significant interaction between drug class and age at baseline for suicide $(\mathrm{P}=0.09)$, although numbers were small. In a subgroup analysis split by age band the rates of suicide were lower for tricyclic antidepressants and other antidepressants compared with selective serotonin reuptake inhibitors in patients aged 20 to 29 (table 4), but these were not statistically significant. In patients aged 40 to 49 and 50 to 64 , the adjusted hazard ratios were significantly increased for other antidepressants compared with selective serotonin reuptake inhibitors.

There was some indication of an interaction between drug class and age for attempted suicide or self harm $(\mathrm{P}=0.02)$. Adjusted hazard ratios were similar for tricyclic antidepressants compared with selective serotonin reuptake inhibitors in all age bands, but increased slightly with age for other antidepressants compared with selective serotonin reuptake inhibitors, from 1.62 (1.33 to 1.97 ) in patients aged $20-29$ to 2.29 (1.75 to 3.00) in patients aged 50-64.

\section{Absolute risks of suicide and attempted suicide or self harm}

Table 5 shows the absolute risks of suicide and attempted suicide or self harm over one year. For suicide 
Table 3 | Unadjusted and adjusted hazard ratios for attempted suicide or self harm by antidepressant class, dose, and individual drug over five year follow-up period

\begin{tabular}{|c|c|c|c|c|c|c|}
\hline \multirow[b]{2}{*}{ Variables } & \multicolumn{3}{|c|}{ Unadjusted analysis } & \multirow[b]{2}{*}{$P$ value } & \multicolumn{2}{|l|}{ Adjusted analysis* } \\
\hline & No of eventst & Person yearst & $\begin{array}{l}\text { Hazard ratio } \\
(95 \% \mathrm{Cl})\end{array}$ & & $\begin{array}{l}\text { Hazard ratio }(95 \% \\
\mathrm{Cl})\end{array}$ & Pvalue \\
\hline \multicolumn{7}{|l|}{ Antidepressant class } \\
\hline SSRIS & 2357 & 215665 & 1.00 & & 1.00 & \\
\hline TCAs & 400 & 39444 & 0.94 (0.84 to 1.04$)$ & 0.2 & 0.96 (0.87 to 1.08$)$ & 0.5 \\
\hline Other antidepressants & 483 & 25646 & 2.01 (1.81 to 2.23$)$ & $<0.001$ & 1.80 (1.61 to 2.00$)$ & $<0.001$ \\
\hline Combined antidepressants & 68 & 3803 & 2.14 (1.67 to 2.74$)$ & $<0.001$ & 2.00 (1.54 to 2.59$)$ & $<0.001$ \\
\hline No current use & 1741 & 545180 & 0.40 (0.37 to 0.43$)$ & $<0.001$ & 0.36 (0.34 to 0.39$)$ & $<0.001$ \\
\hline \multicolumn{7}{|c|}{ Antidepressant class and dose categories (defined daily doses)‡ } \\
\hline \multicolumn{7}{|l|}{ SSRIs: } \\
\hline$\leq 0.5$ & 100 & 15713 & $0.70(0.57$ to 0.85$)$ & 0.001 & 0.79 (0.64 to 0.97$)$ & 0.03 \\
\hline$>0.5 / \leq 1.0$ & 1550 & 151769 & 1.00 & & 1.00 & \\
\hline$>1.0$ & 617 & 39742 & 2.07 (1.88 to 2.29$)$ & $<0.001$ & 1.91 (1.73 to 2.11$)$ & $<0.001$ \\
\hline Test for trend§ & & & & $<0.001$ & & $<0.001$ \\
\hline \multicolumn{7}{|l|}{ TCAs: } \\
\hline$\leq 0.5$ & 211 & 22712 & 0.96 (0.83 to 1.11$)$ & 0.6 & $1.03(0.89$ to 1.20$)$ & 0.6 \\
\hline$>0.5 / \leq 1.0$ & 94 & 7977 & 1.33 (1.06 to 1.66$)$ & 0.01 & 1.27 (1.01 to 1.60$)$ & 0.04 \\
\hline$>1.0$ & 60 & 4935 & 1.35 (1.04 to 1.75$)$ & 0.02 & 1.21 (0.93 to 1.58$)$ & 0.1 \\
\hline Test for trend§ & & & & $<0.001$ & & 0.01 \\
\hline \multicolumn{7}{|l|}{ Others: } \\
\hline$\leq 0.5$ & 52 & 3793 & 1.65 (1.27 to 2.14$)$ & $<0.001$ & 1.63 (1.24 to 2.13$)$ & $<0.001$ \\
\hline$>0.5 / \leq 1.0$ & 213 & 12144 & 2.11 (1.82 to 2.45$)$ & $<0.001$ & 1.88 (1.61 to 2.19$)$ & $<0.001$ \\
\hline$>1.0$ & 189 & 7440 & 3.68 (3.18 to 4.26$)$ & $<0.001$ & 3.09 (2.65 to 3.60$)$ & $<0.001$ \\
\hline Test for trend§ & & & & $<0.001$ & & $<0.001$ \\
\hline \multicolumn{7}{|l|}{ Antidepressant drugs } \\
\hline \multicolumn{7}{|l|}{ SSRIS: } \\
\hline Citalopram & 975 & 90494 & 1.00 & & 1.00 & \\
\hline Escitalopram & 144 & 12716 & 1.06 (0.89 to 1.27$)$ & 0.5 & 1.05 (0.87 to 1.26$)$ & 0.6 \\
\hline Fluoxetine & 834 & 78458 & 0.95 (0.86 to 1.04$)$ & 0.3 & 0.92 (0.84 to 1.02 ) & 0.1 \\
\hline Paroxetine & 190 & 15851 & 1.07 (0.93 to 1.24$)$ & 0.3 & 0.91 (0.78 to 1.06$)$ & 0.2 \\
\hline Sertraline & 213 & 17884 & 1.15 (1.00 to 1.32$)$ & 0.05 & 1.07 (0.92 to 1.23$)$ & 0.4 \\
\hline \multicolumn{7}{|l|}{ TCAs: } \\
\hline Amitriptyline & 133 & 18818 & 0.68 (0.57 to 0.81$)$ & $<0.001$ & 0.71 (0.59 to 0.85$)$ & $<0.001$ \\
\hline Dosulepin & 138 & 11690 & 1.03 (0.85 to 1.24$)$ & 0.8 & 1.04 (0.86 to 1.26$)$ & 0.7 \\
\hline Lofepramine & 62 & 4500 & 1.18 (0.93 to 1.50$)$ & 0.2 & 1.08 (0.84 to 1.38$)$ & 0.6 \\
\hline Trazodone & 45 & 2136 & 2.03 (1.48 to 2.78$)$ & $<0.001$ & $1.73(1.26$ to 2.37$)$ & 0.001 \\
\hline \multicolumn{7}{|l|}{ Others: } \\
\hline Mirtazapine & 178 & 9120 & 2.02 (1.72 to 2.38$)$ & $<0.001$ & 1.70 (1.44 to 2.02$)$ & $<0.001$ \\
\hline Venlafaxine & 276 & 14440 & 2.09 (1.82 to 2.40$)$ & $<0.001$ & 1.85 (1.61 to 2.13$)$ & $<0.001$ \\
\hline All other antidepressants & 52 & 4648 & 1.17 (0.90 to 1.52$)$ & 0.3 & 1.05 (0.80 to 1.39$)$ & 0.7 \\
\hline Combined antidepressants & 68 & 3803 & 2.14 (1.67 to 2.74$)$ & $<0.001$ & $1.94(1.50$ to 2.52$)$ & $<0.001$ \\
\hline
\end{tabular}

SSRIs=selective serotonin reuptake inhibitors; TCAs=tricyclic and related antidepressants.

*Adjusted for age, sex, year of diagnosis of depression, severity of depression, deprivation, smoking status, alcohol intake, ethnic group, coronary heart disease, diabetes, hypertension, cancer, epilepsy or seizures, hypothyroidism, osteoarthritis, asthma or chronic obstructive airways disease, stroke or transient ischaemic attack, rheumatoid arthritis, osteoporosis, liver disease, renal disease, obsessive-compulsive disorder, statins, non-steroidal anti-inflammatory drugs, aspirin, antihypertensives, anticonvulsants, hypnotics or anxiolytics, antipsychotics, bisphosphonates, anticoagulants, oral contraceptives, and hormone replacement therapy. tBased on numbers in adjusted analysis.

₹Total numbers of events in analysis of dose are reduced owing to missing data on dose.

$\S$ Test for trend uses continuous values of dose.

the absolute risks over one year ranged from $0.02 \%$ for amitriptyline to $0.19 \%$ for mirtazapine. For attempted suicide or self harm the absolute risks ranged from $1.02 \%$ for amitriptyline to $2.96 \%$ for venlafaxine.

\section{Discussion}

In this large cohort study of patients aged 20 to 64 with a diagnosis of depression, we found significant associations between different classes and types of antidepressants and rates of suicide and attempted suicide or self harm. The group of antidepressants classified as "other antidepressants" (mainly comprising venlafaxine and mirtazapine) was associated with the highest rates of both of these outcomes, whereas among individual drugs mirtazapine, venlafaxine, and trazodone were associated with increased risks of attempted suicide or self harm compared with the most commonly prescribed antidepressant, citalopram.

Rates of suicide and attempted suicide or self harm were lowest during periods when patients were not taking antidepressants, but this is likely to reflect the absence of current depression or less severe depression 


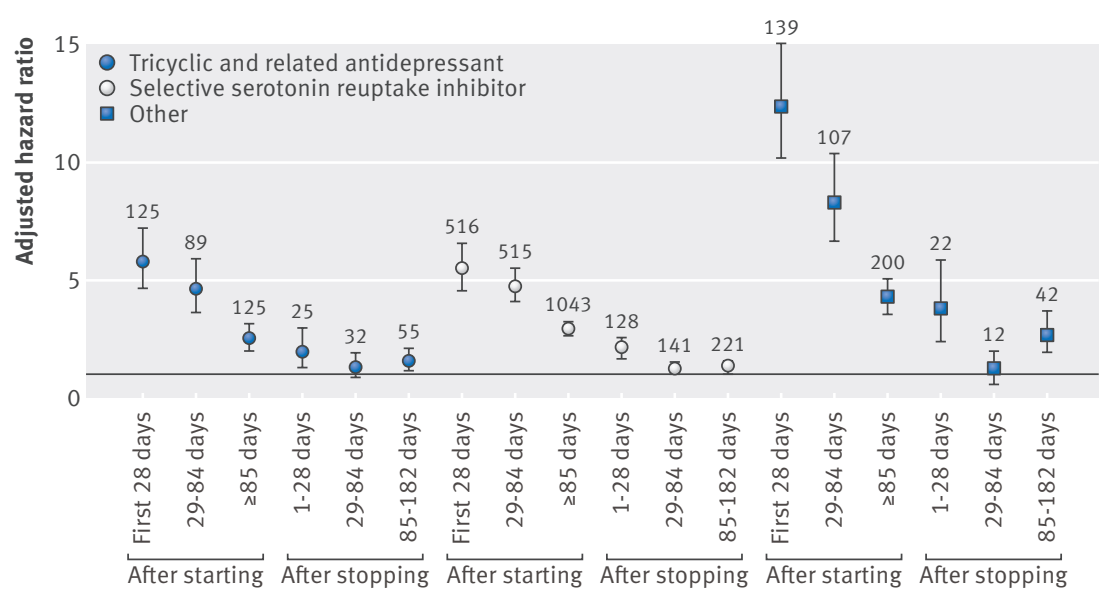

Time since starting and stopping treatment

Fig 4 | Adjusted hazards and $95 \%$ confidence intervals for attempted suicide or self harm according to time since starting and stopping different classes of antidepressant compared with untreated periods. Numbers of events are included

during those periods rather than an indication that antidepressant treatment in itself increases the risks of suicide and attempted suicide or self harm.

The higher hazard ratios for death from suicide in association with venlafaxine and mirtazapine compared with those for attempted suicide or self harm could be due to the higher lethality of these drugs in overdose than selective serotonin reuptake inhibitor antidepressants, ${ }^{22}$ but this would not explain their higher hazard ratios compared with tricyclic antidepressants, which have much higher toxicity. Also numbers of suicide events were small, so these results should be interpreted with caution.

\section{Strengths and limitations of this study}

This study was large and population based with broad inclusion criteria. We included all eligible patients in the study, so the findings are generalisable to people aged 20 to 64 years with a diagnosis of depression in primary care. Recall bias is not an issue as we used data on prescriptions for antidepressants and potential confounding variables, which were prospectively recorded before the occurrence of the outcomes. We had detailed information on prescriptions for antidepressants issued in primary care throughout the follow-up period, so we were able to investigate associations with individual drugs, dose, and duration of treatment.

\begin{tabular}{|c|c|c|c|c|c|c|}
\hline \multirow{2}{*}{$\begin{array}{l}\text { Antidepressant class } \\
\text { and age groups }\end{array}$} & \multicolumn{3}{|l|}{ Suicide } & \multicolumn{3}{|c|}{ Attempted suicide or self harm } \\
\hline & No of events* & Hazard ratiot $(95 \% \mathrm{Cl})$ & Pvalue & No of events* & Hazard ratio $\neq(95 \% \mathrm{Cl})$ & Pvalue \\
\hline \multicolumn{7}{|l|}{ SSRIs: } \\
\hline $20-29$ & 11 & 1.00 & - & 743 & 1.00 & - \\
\hline $30-39$ & 23 & 1.00 & - & 792 & 1.00 & - \\
\hline $40-49$ & 27 & 1.00 & - & 567 & 1.00 & - \\
\hline $50-64$ & 18 & 1.00 & - & 255 & 1.00 & - \\
\hline \multicolumn{7}{|l|}{ TCAs: } \\
\hline $20-29$ & 1 & 0.55 (0.08 to 3.59$)$ & 0.5 & 111 & $1.00(0.80$ to 1.24$)$ & 1.0 \\
\hline $30-39$ & 1 & 0.21 (0.03 to 1.55$)$ & 0.1 & 124 & 0.93 (0.77 to 1.12$)$ & 0.4 \\
\hline $40-49$ & 5 & 1.11 (0.43 to 2.90$)$ & 0.8 & 95 & 0.87 (0.70 to 1.07$)$ & 0.2 \\
\hline $50-64$ & 6 & 1.40 (0.54 to 3.59$)$ & 0.5 & 70 & 1.18 (0.89 to 1.55$)$ & 0.2 \\
\hline \multicolumn{7}{|l|}{ Others: } \\
\hline $20-29$ & 1 & 0.50 (0.07 to 3.77$)$ & 0.5 & 124 & $1.62(1.33$ to 1.97$)$ & $<0.001$ \\
\hline $30-39$ & 5 & 1.27 (0.46 to 3.51$)$ & 0.6 & 160 & 1.74 (1.45 to 2.10$)$ & $<0.001$ \\
\hline $40-49$ & 10 & 2.79 (1.37 to 5.64$)$ & 0.004 & 124 & $1.82(1.50$ to 2.21$)$ & $<0.001$ \\
\hline $50-64$ & 15 & 5.55 (2.73 to 11.29$)$ & $<0.001$ & 75 & 2.29 (1.75 to 3.00$)$ & $<0.001$ \\
\hline \multicolumn{7}{|l|}{ No current use: } \\
\hline $20-29$ & 17 & $0.32(0.13$ to 0.77$)$ & 0.01 & 670 & 0.36 (0.32 to 0.42$)$ & $<0.001$ \\
\hline $30-39$ & 20 & 0.30 (0.15 to 0.59$)$ & 0.001 & 592 & 0.35 (0.31 to 0.40$)$ & $<0.001$ \\
\hline $40-49$ & 13 & 0.27 (0.13 to 0.56$)$ & $<0.001$ & 331 & 0.34 (0.29 to 0.40$)$ & $<0.001$ \\
\hline $50-64$ & 19 & 0.64 (0.32 to 1.28$)$ & 0.2 & 148 & 0.41 (0.33 to 0.52$)$ & $<0.001$ \\
\hline
\end{tabular}

SSRIs=selective serotonin reuptake inhibitors; TCAs=tricyclic and related antidepressants.

Results not shown for combined antidepressant treatment.

*Based on numbers in adjusted analysis.

tAdjusted for confounders listed in table 2 .

$\ddagger$ Adjusted for confounders listed in table 3 . 


\begin{tabular}{|c|c|c|}
\hline \multirow[b]{2}{*}{$\begin{array}{l}\text { Drug class and individual } \\
\text { drugs }\end{array}$} & \multicolumn{2}{|c|}{ Absolute risks $(95 \% \mathrm{Cl})$ over one year (\%) } \\
\hline & Suicide* & $\begin{array}{l}\text { Attempted suicide or } \\
\text { self harmt }\end{array}$ \\
\hline \multicolumn{3}{|l|}{ Antidepressant classes } \\
\hline SSRIS & 0.05 (0.01 to 0.11$)$ & 1.38 (1.17 to 1.61$)$ \\
\hline TCAs & 0.04 (0.03 to 0.09$)$ & $1.37(1.24$ to 1.54$)$ \\
\hline Other antidepressants & $0.17(0.08$ to 0.36$)$ & 2.70 (2.28 to 3.20$)$ \\
\hline Combined antidepressants & $0.11(0.02$ to 0.77$)$ & 2.97 (1.91 to 4.62) \\
\hline \multicolumn{3}{|l|}{ Antidepressant drugs } \\
\hline \multicolumn{3}{|l|}{ SSRIS: } \\
\hline Citalopram & 0.05 (0.02 to 0.09$)$ & $1.42(1.25$ to 1.61$)$ \\
\hline Escitalopram & 0.05 (0.02 to 0.19$)$ & $1.61(1.28$ to 2.04$)$ \\
\hline Fluoxetine & 0.04 (0.02 to 0.09$)$ & 1.30 (1.14 to 1.48$)$ \\
\hline Paroxetine & 0.08 (0.03 to 0.21$)$ & 1.40 (1.14 to 1.71$)$ \\
\hline Sertraline & $0.04(0.01$ to 0.14$)$ & 1.41 (1.15 to 1.74$)$ \\
\hline \multicolumn{3}{|l|}{ TCAs: } \\
\hline Amitriptyline & 0.02 (0.00 to 0.13$)$ & $1.02(0.80$ to 1.31$)$ \\
\hline Dosulepin & 0.05 (0.01 to 0.20$)$ & 1.51 (1.19 to 1.92$)$ \\
\hline Lofepramine & $0.05(0.01$ to 0.37$)$ & $1.54(1.12$ to 2.10$)$ \\
\hline Trazodone & 0.10 (0.01 to 0.79$)$ & 2.64 (1.69 to 4.13) \\
\hline \multicolumn{3}{|l|}{ Others: } \\
\hline Mirtazapine & 0.19 (0.07 to 0.47$)$ & 2.55 (2.03 to 3.21$)$ \\
\hline Venlafaxine & $0.14(0.05$ to 0.37$)$ & $2.96(2.40$ to 3.64$)$ \\
\hline All other antidepressants & 0.12 (0.03 to 0.53$)$ & $1.63(1.11$ to 2.41$)$ \\
\hline Combined antidepressants & $0.11(0.02$ to 0.77$)$ & 2.97 (1.91 to 4.62$)$ \\
\hline
\end{tabular}

Although all the participants had a diagnosis of depression, no specific studies have been carried out into the validity and reliability of diagnosis data for depression recorded by doctors in primary care contributing to the QResearch database. However, there is evidence from the United Kingdom and the Netherlands that the diagnosis of depression by general practitioners is valid and reliable. ${ }^{23} 24$

Drug safety can be assessed through meta-analyses of trials reporting adverse outcomes, ${ }^{7}$ but in the case of antidepressants and suicide or attempted suicide these trials tend to be short term and generally exclude patients with any major suicide risk or with physical or psychiatric comorbidity. Indication bias is the main concern with observational studies assessing drug safety, which occurs when patients are prescribed drugs for a condition that is itself associated with the risk of the adverse outcome. To reduce this bias we only included patients with a recorded diagnosis of depression, so that patients had the same indication for treatment. This is important, as depression is associated with a substantially increased risk of suicide and self harm. ${ }^{12}$

Direct comparisons between groups receiving different treatments for the same condition are still subject to channelling bias, where the selection of a particular antidepressant depends on the characteristics of the patient. This could have occurred in this study if drugs such as mirtazapine, venflaxine, and trazodone were more likely to be prescribed to patients at increased risk of suicide or attempted suicide. This may apply to venlafaxine, particularly since guidelines from the National
Institute for Health and Care Excellence suggest switching people with depression that does not respond well to initial treatment firstly to a different selective serotonin reuptake inhibitor or a better tolerated newer generation antidepressant and subsequently to an antidepressant of a different drug class-for example, venlafaxine, a tricyclic antidepressant, or a monoamine oxidase inhibitor. ${ }^{25}$ To reduce the effect of channelling bias we adjusted for a range of potential confounding factors that could influence the choice of antidepressant; for example, in the analysis of suicide we adjusted for attempted suicide at baseline as well as for many other confounders, including severity of depression. However, we could only use a basic measure of the severity of the initial diagnosis of depression based on Read codes, as depression severity scores are not routinely recorded in general practice. The Read codes used to record depression were generally not specific in terms of severity. Also we were not able to account for changes in severity of depression during follow-up since the severity is not recorded consistently over time, so we could only include it in the analysis as a single baseline variable rather than a time varying variable; this means that the severity of depression is likely to still have a confounding influence on our results. In addition there may be other potential confounding variables, such as drug misuse or family history of psychiatric disorder, ${ }^{26}$ which were either not recorded on the database or not recorded in sufficient detail for their confounding effect to be completely removed by analysis. Differences in characteristics between patients prescribed different antidepressants could therefore account for some of the associations we found between the drugs and suicide and self harm, and we cannot therefore exclude the possibility of residual confounding.

Misclassification of drug use may have occurred, as patients might not have taken their prescribed antidepressant. This may particularly be the case for the $14 \%$ of patients who only received a single prescription. We cannot be certain of the exact dates when patients started and stopped taking their prescribed drug. Since we did not have a precise stopping date we assumed patients to be exposed to an antidepressant where there were no gaps of more than 90 days between the end of one prescription and the start of the next, to allow for any antidepressant use if patients had accumulated extra tablets over time and also so that outcomes occurring during withdrawal periods would be attributed to the antidepressant. This misclassification could underestimate associations with drug use if non-differential but could exaggerate the associations, since patients missing antidepressants for only a few days or weeks may have substantial changes in mood and suicidal ideation. When we changed the gap to 30 days, however, the results were similar. The increased rates we found during periods shortly after antidepressants were started might be a consequence of patients switching between treatments, making these associations difficult to disentangle.

Some outcomes may also have been misclassified, especially where episodes of attempted suicide or self 
harm are not reported. This could lead to underestimation of absolute risks but is unlikely to vary according to the antidepressant prescribed so this would not explain the associations we have found. We included information from linked death certificates to identify additional cases of suicide, including those recorded as open verdicts, which will have reduced misclassification for this outcome; however, we might still have underestimated suicide rates since some suicides may have been recorded as unintentional incidents. ${ }^{27}$

The numbers of events in the suicide analyses were small, particularly when split into different antidepressant exposure categories. So while there was sufficient power to detect large differences, such as between other antidepressants and selective serotonin reuptake inhibitors (adjusted hazard ratio 2.6, 95\% confidence interval 1.7 to 4.0 ), the power would have been low for smaller differences. Therefore we cannot rule out relatively small differences between, for example, selective serotonin reuptake inhibitors and tricyclic antidepressants. Furthermore the small number of suicide events means that the multivariable models may be overfitted, so for individual drugs in particular the adjusted hazard ratios and absolute event rates derived from them should be interpreted with extreme caution.

A further limitation is that the underlying biology of suicide and attempted suicide is poorly understood so there is no established pharmacological rationale for the relations we have identified; rather, these are statistical associations worthy of further investigation to understand any underlying biological mechanisms.

\section{Comparison with other studies}

The suicide rates in our study cohort (43 per 100000 in men and 9 per 100000 in women) are higher than those in the general population in England ${ }^{28}$ (three year average rates of 12.4 per 100000 in men and 3.7 per 100000 in women for 2010-12). Larger differences than this might be expected since our rates are in patients with a diagnosis of depression rather than in the general population. Studies showing greater differences, however, have tended to be in secondary care settings, where patients have more severe depression.

These results are consistent with those of our previous cohort study in older people with depression, ${ }^{19}$ which found that trazodone, mirtazapine, and venlafaxine were associated with the highest rates of attempted suicide or self harm in people aged 65 or more. The number of recorded suicides in that study was too small ( 43 cases) to carry out an analysis by individual drug, but the analysis by drug class found that the suicide rate was highest for the group of other antidepressants.

Some other observational studies of antidepressants and risk of suicide or attempted suicide have also found increased risks among patients prescribed venlafaxine, although fewer studies have reported increased risks for mirtazapine or trazodone. A cohort study ${ }^{9}$ in patients aged 18 to 89 using the general practice research database found that venlafaxine use was associated with a higher risk of suicide and attempted suicide than was citalopram, fluoxetine, and dothiepin. The authors concluded that this could reflect residual confounding since they found that venlafaxine users had a higher burden of risk factors for suicide, as was also the case in a previous study using the general practice research database..$^{29}$ A study of US veterans ${ }^{30}$ with diagnosed depression comparing seven antidepressant medications found that crude suicide rates were highest among those prescribed mirtazapine, and venlafaxine had the second highest crude rate. In adjusted analyses, however, the suicide rates were not significantly higher for these drugs than for citalopram. A cohort study comprising all patients in Finland admitted to hospital because of a suicide attempt between 1997 and 2003, excluding patients with a diagnosis of psychosis, found that venlafaxine was associated with a significantly increased risk of suicide during follow-up and fluoxetine with a significantly reduced risk when compared with no antidepressant treatment, even after adjusting for confounders. ${ }^{31}$ Mirtazapine was associated with an increased risk of suicide in the crude analysis, but not after adjustment. In the adjusted analysis all individual antidepressant drugs were associated with an increased risk of further suicide attempts compared with no antidepressant use, and although the adjusted relative risk was highest for venlafaxine it was only slightly larger than the relative risks for the other antidepressants studied. A cohort study ${ }^{10}$ based on incident users of antidepressants also found a significantly increased risk of suicide acts (suicide attempts and completed suicides) for venlafaxine users compared with selective serotonin reuptake inhibitor users, although the association was attenuated in a secondary analysis restricted to treatment naïve patients.

Although randomised controlled trials of antidepressants are not influenced by residual confounding, they tend to be small, of short duration, and often exclude people who are actively suicidal or have comorbidities. ${ }^{32}$ Several meta-analyses of randomised controlled trials have been conducted, ${ }^{73-36}$ although few have looked at individual antidepressant drugs. The meta-analysis of 372 randomised controlled trials conducted by Stone et $\mathrm{al}^{7}$ included an analysis of suicidality risk (suicide, attempted suicide, preparatory acts, or ideation) for 18 individual drugs compared with placebo. This meta-analysis included a total of 530 outcome events, of which only eight were completed suicides and 134 were attempted suicides. The numbers of events for individual drugs were small and statistical tests found no significant differences among drugs and drug classes, with the exception of some indication of differences among selective serotonin reuptake inhibitors, where odds ratios for suicidality ranged from 0.51 ( $95 \%$ confidence interval 0.29 to 0.91 ) for sertraline to 2.44 (0.90 to 6.63) for escitalopram compared with placebo.

\section{Clinical implications and future research}

The results of this study and others on self harm, suicide, and all cause mortality increasingly suggest that adults prescribed drugs such as mirtazapine and 
venlafaxine and possibly trazodone seem to be at higher risk of self harm and suicide. These associations might be due to these patients having different characteristics or more severe depression than patients prescribed other antidepressants rather than a direct causal effect of the drugs, but irrespective of the reason for the increased risks these patients need particularly close monitoring during treatment and shortly after stopping. The benefits and risks of different antidepressant treatments will vary between patients, ${ }^{37}$ and these should be considered carefully when antidepressants are prescribed.

At present NICE guidelines for depression ${ }^{25}$ recommend the use of a selective serotonin reuptake inhibitor first but if there is a lack of response to use another selective serotonin reuptake inhibitor or a better tolerated newer generation antidepressant, and subsequently an antidepressant from a different drug class, such as venlafaxine or a tricyclic antidepressant. The British Association of Psychopharmacology guidelines $^{38}$ recommend the use of venlafaxine, escitalopram, or older tricyclic antidepressants at a higher dose in patients with severe depression. If our findings are confirmed this advice may require further consideration. Our results, showing an association of an increased risk of attempted suicide or self harm with combinations of antidepressants compared with selective serotonin reuptake inhibitors is, in addition to adverse physical outcomes, another reason for careful monitoring of patients taking combinations of antidepressants by specialists in the management of depression. ${ }^{25}$ The results also confirm that the risk of self harm and suicide is increased in the first 28 days of stopping antidepressants, so guidelines might place greater emphasis on the need for monitoring anyone after a planned withdrawal of antidepressants or an indication of any unplanned or sudden withdrawal. Further research might examine if such risk is universal or only applies to patients with symptoms of depression and other risk factors for suicide and self harm.

\section{Conclusions}

This study has found that rates of suicide and self harm were similar during periods of treatment with selective serotonin reuptake inhibitors and tricyclic antidepressants, but were higher for the group of other antidepressant drugs, with mirtazapine, venlafaxine, and trazodone being associated with the highest risks. The number of suicide events was small so the results for suicide should be interpreted with caution. Rates tended to be highest in the first 28 days after starting treatment and remained increased in the first 28 days after stopping treatment. These findings are of associations rather than causal effects and are particularly susceptible to confounding by indication, channelling bias, and residual confounding; further research is needed to confirm them. The results of this study indicate that patients taking antidepressant drugs should be carefully monitored, especially during early treatment with antidepressants and when stopping treatment.
We thank those practices who use EMIS (Egton Medical Information Systems) and contribute to QResearch, and we thank EMIS and the University of Nottingham for expertise in establishing, developing, and supporting the database.

Contributors: CC, JH-C, RM, AA, and MM contributed to the overal conception and design of the study. CC wrote the first draft of this manuscript. JH-C undertook the data extraction. $\mathrm{TH}$ and $\mathrm{CC}$ carried out the statistical analyses of the study. All authors contributed to the interpretation of results and drafting of this manuscript. All authors read and approved the final manuscript. The views expressed are those of the authors and not necessarily those of the National Health Service, the NIHR, or the Department of Health. CC is the guarantor.

Funding: This project was funded by the National Institute for Health Research (NIHR) School for Primary Care Research (project No 81). RM's contribution to the study has been funded through the NIHR Collaboration for Leadership in Applied Health Research and Care East Midlands (CLAHRC EM). The funding body did not play a role in the study design, writing of the manuscript, or the decision to submit the manuscript for publication

Competing interests: All authors have completed the ICMJE uniform disclosure form at www.icmje.org/coi_disclosure.pdf and declare: financial support from NIHR for the submitted work; JH-C is director of QResearch, which is a not for profit venture between the University of Nottingham and Egton Medical Information Systems (commercial supplier of general practice clinical systems); no financial relationships with any organisations that might have an interest in the submitted work in the previous three years; no other relationships or activities that could appear to have influenced the submitted work.

Ethical approval: The project has been independently peer reviewed and accepted by the QResearch Scientific board and has been approved in accordance with the agreed procedure with the Trent Research Ethics Committee (reference No MREC/03/4/021).

Data sharing: The patient level data from the QResearch are specifically licensed according to its governance framework. See www. qresearch.org for further details.

Transparency: The lead author (CC) affirms that this manuscript is an honest, accurate, and transparent account of the study being reported; that no important aspects of the study have been omitted; and that any discrepancies from the study as planned have been explained.

This is an Open Access article distributed in accordance with the Creative Commons Attribution Non Commercial (CC BY-NC 4.0) license, which permits others to distribute, remix, adapt, build upon this work non-commercially, and license their derivative works on different terms, provided the original work is properly cited and the use is non-commercial. See: http://creativecommons.org/licenses/ by-nc/4.0/.

Harris EC, Barraclough B. Suicide as an outcome for mental disorders. A meta-analysis. Br J Psychiatry 1997;170:205-28.

2 Cassano P, Fava M. Depression and public health: an overview. J Psychosom Res 2002;53:849-57.

3 Arroll B, Elley CR, Fishman T, Goodyear-Smith FA, Kenealy T, Blashki G, et al. Antidepressants versus placebo for depression in primary care. Cochrane Database Syst Rev 2009;3:CD007954.

4 Gibbons RD, Hur K, Brown C, Davis JM, Mann J. Benefits from antidepressants: synthesis of 6-week patient-level outcomes from double-blind placebo-controlled randomized trials of fluoxetine and venlafaxine. Arch Gen Psychiatry 2012;69:572-9.

5 Friedman RA, Leon AC. Expanding the black box-depression, antidepressants, and the risk of suicide. N Engl J Med 2007;356:2343-6.

6 Hall WD. How have the SSRI antidepressants affected suicide risk? Lancet 2006;367:1959-62.

7 Stone M, Laughren T, Jones ML, Levenson M, Holland PC, Hughes A, et al. Risk of suicidality in clinical trials of antidepressants in adults: analysis of proprietary data submitted to US Food and Drug Administration. BM/ 2009;339:b2880.

8 Barbui C, Esposito E, Cipriani A. Selective serotonin reuptake inhibitors and risk of suicide: a systematic review of observational studies. CMAl 2009;180:291-7.

9 Rubino A, Roskell N, Tennis P, Mines D, Weich S, Andrews E. Risk of suicide during treatment with venlafaxine, citalopram, fluoxetine, and dothiepin: retrospective cohort study. BMJ 2007;334:242.

10 Schneeweiss S, Patrick AR, Solomon DH, Mehta J, Dormuth C, Miller M, et al. Variation in the risk of suicide attempts and completed suicides by antidepressant agent in adults: a propensity score-adjusted analysis of 9 years' data. Arch Gen Psychiatry 2010;67:497-506.

11 Coupland C, Dhiman P, Morriss R, Arthur A, Barton G, Hippisley-Cox J. Antidepressant use and risk of adverse outcomes in older people: population based cohort study. BMJ 2011;343:d4551. 
12 Office for National Statistics. Suicides in the United Kingdom, 2011 ONS, 2013.

13 Barr B, Taylor-Robinson D, Scott-Samuel A, McKee M, Stuckler D. Suicides associated with the 2008-10 economic recession in England: time trend analysis. BMJ 2012;345:e5142.

14 Chang S-S, Stuckler D, Yip P, Gunnell D. Impact of 2008 global economic crisis on suicide: time trend study in 54 countries. BMJ 2013;347:f5239.

15 Coupland C, Morriss R, Arthur A, Moore M, Hill T, Hippisley-Cox Safety of antidepressants in adults aged under 65: protocol for a cohort study using a large primary care database. BMC Psychiatry 2013;13:135.

16 Martinez C, Rietbrock S, Wise L, Ashby D, Chick J, Moseley J, et al. Antidepressant treatment and the risk of fatal and non-fatal self harm in first episode depression: nested case-control study. BMJ 2005;330:389.

17 Hippisley-Cox J, Pringle M, Hammersley V, Crown N, Wynn A, Meal A, et al. Antidepressants as risk factor for ischaemic heart disease: case-control study in primary care. BMJ 2001;323:666-9.

18 Hall GC. Validation of death and suicide recording on the THIN UK primary care database. Pharmacoepidemiol Drug Saf 2009;18:120-31.

19 Coupland C, Dhiman P, Barton G, Morriss R, Arthur A, Sach T, et al. A study of the safety and harms of antidepressant drugs for older people: a cohort study analysis using a large primary care database. Health Technol Assess 2011:15(28).

20 Williams RL. A note on robust variance estimation for clustercorrelated data. Biometrics 2000;56:645-6.

21 Altman DG, Andersen PK. Calculating the number needed to treat for trials where the outcome is time to an event. BMJ 1999;319:1492-5.

22 Hawton K, Bergen H, Simkin S, Cooper J, Waters K, Gunnell D, et al. Toxicity of antidepressants: rates of suicide relative to prescribing and non-fatal overdose. Br I Psychiatry 2010;196:354-8.

23 Cameron IM, Lawton K, Reid IC. Recognition and subsequent treatment of patients with sub-threshold symptoms of depression in primary care. J Affect Disord 2011;130:99-105.

24 Terluin B, van Hout HPJ, van Marwijk HWJ, Adèr HJ, van der Meer K, de Haan M, et al. Reliability and validity of the assessment of depression in general practice: the short depression interview (SDI). Gen Hosp Psychiatry 2002;24:396-405.

25 National Institute for Health and Care Excellence. Depression in adults: the treatment and management of depression in adults (update). (Clinical guideline 90.) 2009. www.nice.org.uk/guidance/cg90
26 Hawton K, Casañas I Comabella C, Haw C, Saunders K. Risk factors for suicide in individuals with depression: a systematic review. J Affect Disord 2013;147:17-28.

27 Gunnell D, Hawton K, Kapur N. Coroners' verdicts and suicide statistics in England and Wales. BMJ 2011;343:d6030.

28 Department of Health. Statistical update on suicide, 2014. DoH. www. gov.uk/government/publications/suicide-prevention-report

29 Mines D, Hill D, Yu H, Novelli L. Prevalence of risk factors for suicide in patients prescribed venlafaxine, fluoxetine, and citalopram. Pharmacoepidemiol Drug Saf 2005;14:367-72.

30 Valenstein M, Kim HM, Ganoczy D, Eisenberg D, Pfeiffer PN, Downing K, et al. Antidepressant agents and suicide death among US Department of Veterans Affairs patients in depression treatment. J Clin Psychopharmacol 2012;32:346-53.

31 Tiihonen J, Lonnqvist J, Wahlbeck K, Klaukka T, Tanskanen A, Haukka J. Antidepressants and the risk of suicide, attempted suicide, and overall mortality in a nationwide cohort. Arch Gen Psychiatry 2006;63:1358-67.

32 Pompili M, Serafini G, Innamorati M, Ambrosi E, Giordano G, Girardi P, et al. Antidepressants and suicide risk: a comprehensive overview. Pharmaceuticals 2010;3:2861-83.

33 Fergusson D, Doucette S, Glass KC, Shapiro S, Healy D, Hebert P, et al. Association between suicide attempts and selective serotonin reuptake inhibitors: systematic review of randomised controlled trials. BMJ 2005;330:396

34 Gunnell D, Saperia J, Ashby D. Selective serotonin reuptake inhibitors (SSRIs) and suicide in adults: meta-analysis of drug company data from placebo controlled, randomised controlled trials submitted to the MHRA's safety review. BMJ 2005;330:385.

35 Khan A, Khan S, Kolts R, Brown WA. Suicide rates in clinical trials of SSRIs, other antidepressants, and placebo: analysis of FDA reports. Am J Psychiatry 2003;160:790-2.

36 Hammad TA, Laughren TP, Racoosin JA. Suicide rates in short-term randomized controlled trials of newer antidepressants. / Clin Psychopharmacol 2006;26:203-7.

37 Healy D, Whitaker C. Antidepressants and suicide: risk-benefit conundrums. J Psychiatry Neurosci 2003;28:331.

38 Anderson I, Ferrier I, Baldwin R, Cowen P, Howard L, Lewis G, et al. Evidence-based guidelines for treating depressive disorders with antidepressants: a revision of the 2000 British Association for Psychopharmacology guidelines. I Psychopharmacol 2008;22:343-96.

(C) BMJ Publishing Group Ltd 2015 